\title{
RATIONAL SURFACES WITH TOO MANY VECTOR FIELDS
}

\author{
GERALD NEUMAN
}

\begin{abstract}
A method is given for constructing smooth rational surfaces with nonreduced automorphism groups, by a sequence of blow-ups of the projective plane. The technique works in all positive characteristics.
\end{abstract}

1. Introduction. The vector space $V_{X}=H^{0}\left(X, \Theta_{X}\right)$ of regular vector fields on a surface $X$ can be identified with the tangent space to the group scheme Aut $X$ at its identity element. Thus a surface $X$ has a nonreduced automorphism group scheme iff $\operatorname{dim} V_{X}>\operatorname{dim}$ Aut $X$. We wish to describe a method for constructing smooth rational surfaces with this property. Since group schemes in characteristic zero are smooth $[1$, p. 101], this will be possible only for positive characteristics. (In the language of deformation theory, $V_{X}$ may also be identified with the set of infinitesimal automorphisms of $X$ parameterized by the scheme $\operatorname{Spec} k[t] /\left(t^{2}\right)$. If $\operatorname{dim} V_{X}>\operatorname{dim}$ Aut $X$, then $X$ has "obstructed" infinitesimal automorphisms, that is, infinitesimal automorphisms which do not extend to an algebraic family of automorphisms.)

If $X$ is a smooth surface, and $Y$ is the blow-up of $X$ at a smooth point $P$, then the relationship between Aut $X$ and Aut $Y$ can be summarized in two rules.

LEMMA (1.1). The identity component Aut $^{0} Y$ is a closed subgroup of Aut $X$, whose support is the identity component of the subgroup of automorphisms which fix $P$.

LEMMA (1.2). The tangent space $V_{Y}$ is the subspace of $V_{X}$ consisting of the vector fields which vanish at $P$.

Lemma (1.2) is the first step in the proof of Lemma (1.1). It follows from Lemma (3.1) below. If we let $C$ denote the closed subscheme of Aut $X$ representing the functor $C: T \mapsto\{T$-Automorphisms of $X \times T$ fixing the closed subscheme $P \times T\}$, then it is not difficult to show that Aut $^{0} Y$ is isomorphic to the identity component $C^{0}$. Details may be found in [3].

Thus our strategy is to blow up at a point which is fixed by too few automorphisms, or, equivalently, at which too many vector fields vanish.

The projective plane has a reduced automorphism group, namely PGL(2). We will make a series of blowings-up of the plane, each time at a point

Received by the editors January 9, 1978.

AMS (MOS) subject classifications (1970). Primary 14J10, $14 \mathrm{~L} 15$.

Key words and phrases. Rational surface, automorphism group scheme, regular vector field.

(C) 1979 American Mathematical Society 0002-9939/79/0000-0400/\$02.75 
infinitely near the previous point, that is, on the new exceptional divisor. It is convenient to use multi-projective coordinates to describe such a procedure.

Let $B_{0}$ denote the projective plane $\mathrm{P}^{2}$ with coordinates $\left(X_{0}, X_{1}, X_{2}\right)$.

Let $P_{0}$ be the point $(1,0,0)$. We can also take inhomogeneous coordinates $x_{1}=X_{1} / X_{0}$ and $x_{2}=X_{2} / X_{0}$ at $P_{0}$. The blow-up $B_{1}$ of the surface $B_{0}$ at the point $P_{0}$ can be viewed as the subvariety of $B_{0} \times \mathbf{P}^{1}$ consisting of all points $\left(X_{0}, X_{1}, X_{2} ; Y_{0}, Y_{1}\right)$ for which $X_{1} Y_{1}=X_{2} Y_{0}$. In inhomogeneous coordinates, with $y_{1}=Y_{1} / Y_{0}$, this gives the familiar local equation $x_{1} y_{1}=x_{2}$.

Choose a point $P_{1}=\left(1,0,0 ; 1, \lambda_{1}\right)$ on the exceptional divisor. The blow-up of $B_{1}$ at this point consists of all points of $B_{1} \times \mathbf{P}^{1}$ of the form $\left(X_{0}, X_{1}, X_{2} ; Y_{0}, Y_{1} ; Z_{0}, Z_{1}\right)$ satisfying the equation

$$
X_{1} Y_{0} Z_{1}=\left(Y_{1}-\lambda_{1} Y_{0}\right) Z_{0} X_{0}
$$

Once more, taking the inhomogeneous coordinate $y_{2}=Z_{1} / Z_{0}$ gives the local equation $x_{1} y_{2}=y_{1}-\lambda_{1}$.

Similarly, for any choice of $\lambda_{1}, \ldots, \lambda_{k}$, we can take a point $P_{k}=$ $\left(1,0,0 ; 1, \lambda_{1} ; \cdots ; 1, \lambda_{k}\right)$ on $B_{k}$ and blow up to get a surface $B_{k+1}$ defined in $B_{k} \times \mathbf{P}^{1}$ as the set of all $\left(X_{0}, X_{1}, X_{2} ; Y_{0}, Y_{1} ; \cdots ; V_{0}, V_{1} ; W_{0}, W_{1}\right)$ for which

$$
X_{1} V_{0} W_{1}=\left(V_{1}-\lambda_{k} V_{0}\right) W_{0} X_{0}
$$

and with $y_{k+1}=W_{1} / W_{0}$ and $y_{k}=V_{1} / V_{0}$, this gives the local equation $x_{1} y_{k+1}=y_{k}-\lambda_{k}$. With this notation, we will investigate how Aut $B_{k+1}$ and $\operatorname{dim} H^{0}\left(B_{k+1}, \Theta_{B_{k+1}}\right)$ depend on the choice of $\lambda_{1}, \ldots, \lambda_{k}$. We can take $\lambda_{1}=0$ without loss of generality.

2. Calculation of automorphisms. We wish to compute the dimension of Aut $B_{k+1}$. By Lemma (1.1), it suffices to determine which automorphisms of $B_{k}$ fix the point $P_{k}$. Applying the lemma repeatedly, we can view an element $\alpha$ of the identity component $\mathrm{Aut}^{0} B_{k}$ as an automorphism of $\mathbf{P}^{2}$. If $\alpha$ fixes $\boldsymbol{P}_{k}$, it must send each curve passing through $P_{k}$ to another curve with the same property. This condition can be stated on $\mathbf{P}^{2}$ : we ask that $\alpha$ send each curve whose proper transform on $B_{k}$ passes through $P_{k}$ to another such curve.

Given $\lambda_{1}, \ldots, \lambda_{k}$, we put

$$
F_{k}(T)=\lambda_{2} T^{2}+\cdots+\lambda_{k} T^{k}
$$

If $S_{k}$ denotes Spec $k[t] /\left(t^{k+1}\right)$, we define

$$
f_{k}: S_{k} \rightarrow \mathbf{P}^{2}
$$

by

$$
t \leftrightarrow x_{1}, \quad F_{k}(t) \leftrightarrow x_{2} .
$$

With this notation, a straightforward calculation proves the following lemma.

LEMMA (2.1). Let $C$ be a smooth curve on $\mathbf{P}^{2}$. Then the proper transform of $C$ on $B_{k}$ passes through $P_{k}$ iff $f_{k}$ factors through $C$. 
Thus to fix $\boldsymbol{P}_{k}$, an automorphism of $\mathbf{P}^{2}$ must commute with $f_{k}$ up to an automorphism of $S_{k}$.

We recall that every automorphism $\alpha$ of $\mathbf{P}^{2}$ can be written as a linear map

$$
\alpha:\left(\begin{array}{c}
X_{0} \\
X_{1} \\
X_{2}
\end{array}\right) \rightarrow\left(\begin{array}{lll}
a X_{0}+b X_{1}+c X_{2} \\
d X_{0}+ & e X_{1}+f X_{2} \\
g X_{0}+ & +h X_{1}+i X_{2}
\end{array}\right)
$$

and that two linear maps give the same automorphism if they differ by a scalar. We wish to determine the conditions imposed on the coefficients of the matrix

$$
\left(\begin{array}{lll}
a & b & c \\
d & e & f \\
g & h & i
\end{array}\right)
$$

when we require $\alpha$ to fix $P_{k}$.

To fix $P_{0}$ at all, we must have $d=g=0$. If $\alpha$ also fixes $P_{1}$, it must fix the line $X_{2}=0$, and so $h$ is likewise zero. Then the matrix is invertible and upper triangular, and its diagonal entries $a, e$, and $i$ are all nonzero. We can now eliminate the ambiguity about scalars by choosing $a=1$.

Then $\alpha$ commutes with $f_{k}$ up to an automorphism of $S_{k}$ iff

$$
F_{k}\left(\frac{e u+f F_{k}(u)}{1+b u+c F_{k}(u)}\right) \equiv \frac{i F_{k}(u)}{1+b u+c F_{k}(u)} \quad \bmod u^{k+1}
$$

AN EXAMPLE. Rather than describe the conditions imposed on the entries $b, c, e, f$, and $i$ by equation (2.2) in general, we would like to work out a specific example, which gives us in each positive characteristic one of our rational surfaces with nonreduced automorphism group.

Let the characteristic of the ground field be $p$. Then we will take $\lambda_{1}=\lambda_{2}$ $=\cdots=\lambda_{2 p}=0, \lambda_{2 p+1}=\lambda \neq 0, \lambda_{2 p+2}=\cdots=\lambda_{3 p}=0$, and $\lambda_{3 p+1}=\mu \neq$ 0.

Then the condition that $\alpha$ fix $P_{3 p+1}$ is

$$
\begin{gathered}
\lambda\left(\frac{e u+f \lambda u^{2 p+1}+f \mu u^{3 p+1}}{1+b u+c \lambda u^{2 p+1}+c \mu u^{3 p+1}}\right)^{2 p+1} \\
+\mu\left(\frac{e u+f \lambda u^{2 p+1}+f \mu u^{3 p+1}}{1+b u+c \lambda u^{2 p+1}+c \mu u^{3 p+1}}\right)^{3 p+1} \\
\equiv \frac{i \lambda u^{2 p+1}+i \mu u^{3 p+1}}{1+b u+c \lambda u^{2 p+1}+c \mu u^{3 p+1}} \bmod u^{3 p+2}
\end{gathered}
$$

The congruence breaks into the two equations:

$$
i \lambda=\lambda e^{2 p+1}
$$

and

$$
3 b^{p} i \lambda+u \mu=\mu e^{3 p+1}+b^{p} e^{2 p+1} \lambda
$$


solving simultaneously, the latter yields

$$
\mu e^{2 p+1}\left(1-e^{p}\right)=\lambda e^{2 p+1} b^{p}(1-3) .
$$

Clearly these give two conditions on the coefficients, and the automorphism group thus has dimension three; we will later see that this choice of $\lambda$ 's imposes only one condition on the tangent fields, giving a tangent space of dimension four.

3. Calculation of vector fields. Given our sequence of surfaces $B_{1}, \ldots, B_{k+1}$, we wish to calculate the dimension of $V_{B_{k+1}}=H^{0}\left(B_{k+1}, \Theta_{B_{k+1}}\right)$ by determining inductively which vector fields on $B_{j}$ vanish at $\boldsymbol{P}_{j}$. To do so, we will first take a vector field vanishing at $P_{j-1}$ and find an expression for the vector field to which it lifts on $B_{j}$.

We can take $x_{1}$ and $y_{j-1}-\lambda_{j-1}$ as parameters at the point $P_{j-1}$. Any vector field $\theta_{j-1}$ on $B_{j-1}$ can then be written in the form $f d / d x_{1}+g d / d y_{j-1}$. If it lifts to a vector field $\boldsymbol{\theta}_{j}$ on $\boldsymbol{B}_{j}$, we wish to find an expression for $\boldsymbol{\theta}_{j}$ in the form $F d / d x_{1}+G d / d y_{j}$.

LEMMA (3.1). The vector field $\boldsymbol{\theta}_{j-1}$ lifts to a regular vector field $\boldsymbol{\theta}_{j}$ on $\boldsymbol{B}_{j}$ iff it vanishes at $P_{j-1}$, and then at $P_{j}$ we can write

$$
\theta_{j}=f \frac{d}{d x_{1}}+g_{j} \frac{d}{d y_{j}}
$$

with $g_{j}=\left(1 / x_{1}\right)\left(g-f y_{j}\right)$.

Proof. Locally at $P_{j-1}$, the surface $B_{j-1}$ is an open subset of Spec $k\left[x_{1}, y_{j-1}\right]$, and the blowing-up corresponds to the ring homomorphism

$$
k\left[x_{1}, y_{j-1}\right] \stackrel{\phi}{\rightarrow} k\left[x_{1}, y_{j-1}, y_{j}\right] /\left(y_{j-1}-\lambda_{j-1}-x_{1} y_{j}\right) .
$$

We may view the vector fields as derivations on these rings, and we will let $D_{w}$ denote $d / d w$ on $k\left[x_{1}, y_{j-1}\right]$, and $\Delta_{w}$ denote $d / d w$ on $k\left[x_{1}, y_{j-1}, y_{j}\right] /\left(y_{j-1}\right.$ $\left.-\lambda_{j-1}-x_{1} y_{j}\right)$

Then

$$
D_{x_{1}}=\Delta_{x_{1}} \circ \phi-\frac{y_{j}}{x_{1}} \Delta_{y_{j}} \circ \phi \text { and } D_{y_{j}}=\frac{1}{x_{1}} \Delta_{y_{j}} \circ \phi .
$$

We conclude that $f d / d x_{1}+g d / d y_{j-1}$ must lift to the vector field

$$
f \frac{d}{d x_{1}}+\frac{1}{x_{1}}\left(g-f y_{j}\right) \frac{d}{d y_{j}} \text {. }
$$

A priori, a vector field of this form has coefficients in the function field $k\left(x_{1}, y_{j}\right)$; for $\theta_{j}$ to be regular on our open subset of $B_{j}, x_{1}$ must divide $g-f y_{j}$. This occurs iff $g$ has no constant term, that is, iff $g$ vanishes at $P_{j-1}$. By symmetry, $\theta_{j}$ is regular on all of $B_{j}$ iff both $f$ and $g$ vanish at $P_{j-1}$. That is, $\boldsymbol{\theta}_{j-1}$ must vanish at $\boldsymbol{P}_{j-1}$. This proves the lemma.

(Since the question is local in the étale topology, this also proves Lemma (1.2).) 
We suppose now that $\boldsymbol{\theta}_{j-1}$ vanishes at $\boldsymbol{P}_{j-1}$, and so lifts to $\boldsymbol{\theta}_{j}$ on $\boldsymbol{B}_{j}$. When does $\theta_{j}$ vanish at $\boldsymbol{P}_{j}$ ? The function $f$ already vanishes at $\boldsymbol{P}_{j-1}$, and therefore vanishes on the entire exceptional divisor over $\boldsymbol{P}_{j-1}$. It remains to examine $g_{j}=\left(1 / x_{1}\right)\left(g-f y_{j}\right)$. If we break $g-f y_{j}$ into a sum of homogeneous parts with respect to $x_{1}$,

$$
g-f y_{j}=H_{0}+H_{1} x_{1}+H_{2} x_{1}^{2}+\ldots,
$$

we see that $H_{0}$ must vanish at $P_{j}$, and that $g_{j}$ vanishes at $P_{j}$ iff $H_{1}$ does.

Thus the vector field $\theta_{j}$ vanishes at $P_{j}$ iff the coefficient $H_{1}$ of the part of $x_{1}$-degree one in $g-f y_{j}$ does.

Returning to $B_{0}$, we recall that $H^{0}\left(\mathbf{P}^{2}, \Theta_{\mathbf{P}^{2}}\right)$ is spanned by the fields $X_{i} d / d X_{j}$, with the single relation

$$
X_{0} \frac{d}{d X_{0}}+X_{1} \frac{d}{d X_{1}}+X_{2} \frac{d}{d X_{2}}=0 .
$$

This relation permits us to express $X_{i} d / d X_{0}$ as

$$
X_{i} \frac{d}{d X_{0}}=\frac{X_{i}}{X_{0}} X_{0} \frac{d}{d X_{0}}=-\frac{X_{i} X_{1}}{X_{0}} \frac{d}{d X_{1}}-\frac{X_{i} X_{2}}{X_{0}} \frac{d}{d X_{2}}
$$

Since

$$
X_{0} \frac{d}{d X_{j}}=\frac{d}{d\left(X_{j} / X_{0}\right)}
$$

we can write a basis in parameters at $(1,0,0)$ in the form

$$
\begin{aligned}
& \alpha=X_{0} \frac{d}{d X_{0}}=-x_{1} \frac{d}{d x_{1}}-x_{2} \frac{d}{d x_{2}}, \\
& \beta=X_{1} \frac{d}{d X_{0}}=-x_{1}^{2} \frac{d}{d x_{1}}-x_{1} x_{2} \frac{d}{d x_{2}}, \\
& \gamma=X_{2} \frac{d}{d X_{0}}=-x_{1} x_{2} \frac{d}{d x_{1}}-x_{2}^{2} \frac{d}{d x_{2}}, \\
& \delta=X_{0} \frac{d}{d X_{1}}=\frac{d}{d x_{1}}, \\
& \varepsilon=X_{1} \frac{d}{d X_{1}}=x_{1} \frac{d}{d x_{1}}, \\
& \zeta=X_{2} \frac{d}{d X_{1}}=x_{2} \frac{d}{d x_{1}}, \\
& \eta=X_{0} \frac{d}{d X_{2}}= \\
& \theta=X_{1} \frac{d}{d X_{2}}=
\end{aligned}
$$

The fields $\alpha, \beta, \gamma, \varepsilon, \zeta$, and $\theta$ vanish at $(1,0,0)$ and lift to $B_{1}$; all of these but $\boldsymbol{\theta}$ vanish at $\boldsymbol{P}_{1}$, and lift to $\boldsymbol{B}_{2}$. 
Lemma (3.1) allows us to compute the form a vector field on $\boldsymbol{B}_{j-1}$ takes on $B_{j}$; this recursive procedure also yields an explicit equation. If a vector field has the form $f d / d x_{1}+g_{k} d / d y_{k}$ at $P_{k}$ for each $k$, then the $g_{k}$ are related by the formula $g_{k}=\left(1 / x_{1}\right)\left(g_{k-1}-f y_{k}\right)$, and so

$$
g_{k}=\frac{g_{1}}{x_{1}^{k-1}}-f \sum_{r=2}^{k} \frac{y_{1}}{x_{1}^{k-r+1}} .
$$

Using the defining equation for $y_{j}, y_{j} x_{1}=y_{j-1}-\lambda_{j-1}$, we can compute that

$$
\frac{y_{j}}{x_{1}^{n}}=y_{j+n}+\sum_{m=0}^{n-1} \frac{\lambda_{m+j}}{x_{1}^{n-m}} \text {. }
$$

It then follows that

$$
\sum_{r=2}^{k} \frac{y_{1}}{x_{1}^{k-r+1}}=(k-1) \frac{y_{k}}{x_{1}}+\sum_{m=0}^{k-1}(m-1) \frac{\lambda_{m}}{x_{1}^{k-m+1}} .
$$

Since we are interested in $H_{1} x_{1}$, the term of $x_{1}$-degree one in $g_{k-1}-f y_{k}$, we combine formulae (3.2) and (3.4) in the single equation

$$
\begin{aligned}
g_{k-1}-f y_{k} & =x_{1} g_{k} \\
& =\frac{g_{1}}{x_{1}^{k-2}}-(k-1) f y_{k}-f \sum_{m=1}^{k-1}(m-1) \frac{\lambda_{m}}{x_{1}^{k-m}} .
\end{aligned}
$$

Then by inspection, using (3.3) in the form

$$
y_{1}=x_{1}^{k-1}\left(y_{k}+\sum_{m=0}^{k-2} \frac{\lambda_{m+1}}{x_{1}^{k-m-1}}\right) \text {, }
$$

we can easily fill in the following table.

TABLE (3.6)

\begin{tabular}{cccl} 
Field & $f$ & $g_{1}$ & $H_{1} x_{1}$ (for $k>1$ ) \\
\hline$\alpha$ & $-x_{1}$ & 0 & $(k-1) y_{k} x_{1}$ \\
$\beta$ & $-x_{1}^{2}$ & 0 & $(k-2) \lambda_{k-1} x_{1}$ \\
$\gamma$ & $-x_{1}^{2} y_{1}$ & 0 & $\sum_{m-2}^{k-1}(m-1) \lambda_{m} \lambda_{k-m} x_{1}$ \\
$\varepsilon$ & $x_{1}$ & $-y_{1}$ & $-k y_{k} x_{1}$ \\
$\zeta$ & $x_{1} y_{1}$ & $-y_{1}^{2}$ & $\sum_{m-2}^{k-1} m \lambda_{m} \lambda_{k-m+1} x_{1}$
\end{tabular}

As we have seen, a vector field will vanish at $P_{k}$ iff the associated $H_{1}$ does. We note that the $H_{1}$ terms for $\beta, \gamma$, and $\zeta$ do not depend on the choice of $\lambda_{k}$, and so if these fields vanish for some $P_{k}$ they must vanish on the entire exceptional divisor. The field $\beta$ vanishes on the divisor if either $\lambda_{k-1}=0$, or $k \equiv 2 \bmod p$. On the other hand, $\alpha$ and $\varepsilon$ only vanish for $\lambda_{k}=0$, or $k \equiv 1$ $\bmod p($ for $\alpha$ ), or $k \equiv 0 \bmod p($ for $\varepsilon$ ). In these cases, $\alpha$ and $\varepsilon$ vanish on the entire exceptional divisor.

Suppose $\lambda_{1}, \ldots, \lambda_{n}=0$. Then the fields $\gamma$ and $\zeta$ will vanish on the exceptional divisor for all $k<2 n+1$. 
Applying these observations to the example discussed in $\$ 2$ above, we recall that the only nonzero $\lambda_{k}$ occurred for $k=2 p+1$ and $k=3 p+1$. Thus, $\alpha, \beta, \gamma$, and $\zeta$ all vanish at $P_{k}$ for all $k<3 p+1$, and $V_{B_{3 p+2}}$ has dimension four. But as we saw, Aut $B_{3 p+2}$ has dimension three. This provides the desired example.

4. Alternatives. The methods used to calculate automorphisms and vector fields, although applied here to a specific example, are general enough to describe many others. The simplest variation would be to postpone the second nonzero $\lambda$ to some distant step, also congruent to $1 \bmod p$; the same result will follow. Instead of using the fields $\alpha$ and $\beta$, we could take the only nonzero $\lambda$ 's at steps congruent to $0 \bmod p$, and construct an example using the field $\varepsilon$.

Finally, we can extend the present example until there are no automorphisms left, and still have vector fields. For example, in characteristic 2 , if the only nonzero $\lambda$ 's are at $k=5,7,9,13$, and 17, then equation (2.2) shows that the only automorphism fixing $P_{17}$ is the identity $(e=i=1, b=c=f=0)$, but the vector fields $\alpha$ and $\beta$ both lift to $B_{17}$ and vanish at $P_{17}$ (Of course, characteristic 2 is a little special here, because the expressions $(k-1) \lambda_{k}$ and $(k-2) \lambda_{k-1}$ can only be synchronized in characteristic 2 . Normally, we can only arrange for a single vector field (e.g., $\alpha$ ) to lift to $B_{n}$ with discrete automorphism group.)

\section{REFERENCES}

1. D. Mumford, Abelian varieties, Oxford University Press, Bombay, 1970.

2. A. N. Rudakov and I. R. Shafarevich, Inseparable morphisms of algebraic surfaces, Izv. Akad. Nauk SSSR Ser. Math. 40 (1976), 1269-1307. (Russian)

3. G. Neuman, Extra vector fields on algebraic surfaces, Thesis, Massachusetts Institute of Technology, Cambridge, Mass., 1977.

Current address: 3 Langdon Street, Apartment 3, Cambridge, Massachusetts 02138 